\title{
VARIABLE FRAME LENGTH OF A HIGHER ORDER SPEECH AR ESTIMATION IN A SPEECH ENHANCEMENT SYSTEM.
}

\author{
Josep M.SALAVEDRA*, Enrique MASGRAU**, Asunción MORENO*, Joan ESTARELLAS* \\ * Department of Signal Theory and Communications. Universitat Politècnica de Catalunya. \\ Apdo. 30002.08080-BARCELONA. SPAIN. E-mail: mia@tsc.upc.es \\ ** Department of Electrical Engineering and Computers. Universidad de Zaragoza. \\ María de Luna, 3. 50015-ZARAGOZA. SPAIN
}

\begin{abstract}
We study some speech enhancement algorithms based on the iterative Wiener filtering method due to Lim-Oppenheim [2], where the AR spectral estimation of the speech is carried out using a 2nd-order analysis. But in our algorithms we consider an AR estimation by means of cumulant analysis. This work extends some preceding papers due to the authors, providing a different frame length where AR estimation is done. Information of previous speech frames is used to initiate speech $A R$ modelling of the current frame. Two parameters are introduced to dessign Wiener filter at first iteration of this iterative algorithm. These parameters are the Interframe Factor IF and the Previous Frame Iteration PFI. They allow a very important noise suppression after processing only first iteration of this algorithm, without any appreciable increase of distortion.
\end{abstract}

\section{INTRODUCTION}

It is well known, that many applications of speech processing that show very high perfomance in laboratory conditions degrade dramatically when working in real environments because of low robustness. The solution we propose here concerns to a preprocessing front-end in order to enhance the speech quality by means of a speech parametric modelling insensitive to the noise. The use of HO cumulants for speech AR modelling calculation provides the desirable uncoupling between noise and speech. It is based on the property that for Gaussian processes only, all cumulants of order greater than two are identically zero [1]. Moreover, the non-Gaussian processes presenting a symmetric p.d.f. have null odd-order cumulants. Considering a Gaussian or a symmetric p.d.f. noise (a good approximation of very real environments) and the non-Gaussian characteristic of the speech (principally for the voiced frames) it would be possible to obtain an spectral AR modelling of the speech more independent of the noise by using, e.g., 3rd-order cumulants of noisy speech instead of common 2nd-order cumulant.

\section{ITERATIVE WIENER ALGORITHMS}

In the original Lim-Oppenheim Method [2], noisy speech is enhanced by means of an iterative Wiener filtering that is defined as:

$$
W(\omega)=\frac{P s}{P s+P r}
$$

This work was supported by TIC 92-0800-C05-(04 where $\operatorname{Pr}$ is the spectrum of the noise signal $r(n)$, estimated in non-speech frames, and Ps is a spectrum estimation of the unavailable clean speech signal. So, both speech and noise spectra estimation must be available to design the Wiener filter at every frame (see fig.1). We talk over signal estimation because both signals are not available and only noisy speech signal can be processed.

An iterative Wiener filtering is used to obtain a better estimation of the AR speech modelling:

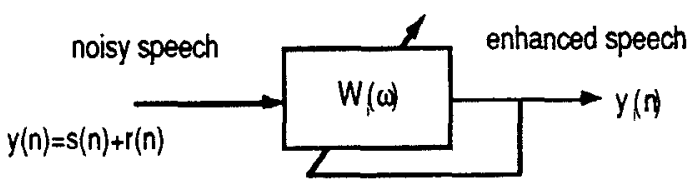

$$
W(\omega)=\frac{P_{Y_{\nu 1}}(\omega)}{P_{Y_{-1}}(\omega)+P_{i}(\omega)} \quad \text { where } \quad P_{y_{\nu 1}}(\omega)=\frac{g^{2}}{\left|1+\sum_{k=1}^{p} a_{k} e^{-\infty \omega)^{2}}\right|^{2}}
$$

Fig.1: Scheme of the iterative Wiener algorithm.

Some different implementations of the iterative Wiener filtering Method based on an AR modelling of the speech signal have been considered. They have been tested under the same algorithm features:

1) segment the noisy speech by using a $50 \%$ overlapping and a frame length of $\mathrm{N}=256$ samples $(32 \mathrm{~ms}$ at $8 \mathrm{kHz}$ sampling frequency).

2) window every frame by Hanning windowing.

3) estimate the noise spectrum inside of non-speech frames by means of a smoothing periodogram.

4) estimate clean speech coefficients of the 10th-order AR modelling from the noisy speech signal.

5) dessign the non-causal Wiener filter from the above estimation of the speech and noise spectra.

6) filter the noisy speech frame through the previously designed Wiener filter. We consider a suitable FFT length in order to avoid aliasing effects caused by circular convolution ( $\mathrm{L}=512$ points $\mathrm{FFT}$ ).

7) itcrate until maximum number of iterations: GO TO stcp 4, by using the filtered speech signal instead of the noisy speech signal to estimate the clean speech spcctrum. 
At first sight an improvement of performance can be expected after every iteration since this current $A R$ speech estimation is carried out from a cleaner speech signal than the filter estimation of the preceding iteration. But other factors sidetrack this iterative algorithm and a limitation in the number of iterations must be taken in account. Clearly the filtered speech signal contains a smaller residual noise but it presents a larger spectral distortion. Therefore, increasing the number of iterations doesn't always involve a better speech estimation. It is well known that this algorithm leads to a narrowness and a shifting of the speech formants [3], providing an unnatural sounding speech. In [4] a detailed convergence analysis of this algorithm is carried out. It is proved that this estimated Wiener filter tends to cancel all signal frequencies with SNR lower than $4.77 \mathrm{~dB}$, and an additional attenuation, proporcionally to the noise level, affects signal frequencies with higher SNR, in comparison to the optimum Wiener filter. Only the non-contaminated speech frequencies undergo a null attenuation.

\section{THE PARAMETERIZED ALGORITHM}

A parameterized Wiener filtering has been considered to have a better control over noise suppression, intelligibility loss and computational complexity, by adding two parameters $\partial$ and $B$ in the Wiener filter computation (1). So, we consider now the following equation:

$$
w_{i}(w)=\left(\frac{P_{y}}{P_{y}+B \cdot P_{r}}\right)^{\partial}
$$

$B y$ varying these parameters $\partial$ and $B$, filters with different characteristics can be obtained. Thus, if $\partial=\beta=1.0$ then expression (2) corresponds to the general Wiener filter equation (1), and if $\partial=0.5, B=1.0$ it corresponds to power spectrum filtering. In [6], a detailed study of performance was performed. High values of both parameters lead to a more aggresive Wiener filter and so noise suppression is increased but distortion increases too. We found that $\partial=1.0, B=1.2$ is a good trade-off among noise suppression, distortion and convergence speed of the iterative filtering, when 3rd-order statistics and low SNR are considered.

AR modelling (fig.1) of the speech spectrum estimation is computed from 3rd-order cumulants that are calculated using the covariance case:

$C_{k}(i, j)=\sum_{n=p+1}^{N} x(n-k) \cdot x(n-i) \cdot x(n-j) \quad, \quad 0 \leq k, i, j \leq p$

where $p=10$ is the order of the filter. Then speech AR modelling coefficients $a_{k}$ are computed by solving the following equations [1]:

$$
\sum_{k=0}^{p} a_{k} \cdot c_{k}(i, j)=0 \quad, 1 \leq i \leq p ; 0 \leq j \leq i
$$

As discussed in preceding works due to the authors $[5],[6]$, we obtain a twofold benefit by considering this 3rd-order AR modelling: Firstly, an accelerated convergence of the iterative algorithm and so a reduction of both computational complexity and intelligibility loss; Secondly, achievement of a non polluted AR speech parameterization. In comparison to 2 nd-order statistics estimation we obtain a good improvement but the price we pay for these advantages is a higher distortion. Thus a higher "peaking" or "narrowness" effect of the speech formants is brought about [4].

When the additive noise is $A W G N$ at $S N R=0 \mathrm{~dB}$ the improvement over second-order algorithm is very appreciated for any number of iterations (see Table 1).

\begin{tabular}{|c|c|c|c|c|c|}
\hline 8 & SNR & SEGSN & IIAKU & COSH & CEPST \\
\hline 0 oten & 0.00 & 0.79 & 9.57 & 11.67 & 12.02 \\
\hline 1 liet & 8.13 & 5.04 & 5.13 & 7.76 & 7.76 \\
\hline $2 \mathrm{Hter}$ & 8.18 & 5.68 & 5.08 & 7.49 & 7.97 \\
\hline 3 iter & 8.05 & 6.03 & 4.84 & 7.28 & 7.93 \\
\hline $4 \mathrm{tten}$ & 7.94 & 6.10 & 4.76 & 7.33 & 7.93 \\
\hline
\end{tabular}
While the improvement of second-order approach increases

\begin{tabular}{|c|c|c|c|r|r|}
\hline d) & SNR & SECSN & TTAKU & COSH & CEPST \\
\hline 0 iter & 0.00 & 0.79 & 9.57 & 11.67 & 12.02 \\
\hline \% iter & 7.47 & 4.53 & 8.97 & 10.49 & 10.53 \\
\hline iter & 7.39 & 4.95 & 7.88 & 9.65 & 9.30 \\
\hline 4 iter & 7.37 & 5.11 & 6.55 & 8.65 & 8.80 \\
\hline
\end{tabular}

Table.1: Distance measures using algorithms based on: a) second order statistic; b) third order cumulants; c) third order with interframe factor $I F=0.6$, considering 5 th itcration of previous frame $(P F I=5)$; $)$ fourth order cumulants at $S N R=0 d B$. 
gradually, but slowly, iteration by iteration, 3rd-order one gets a very good improvement, about $3 \mathrm{~dB}$, after only two iterations and thus it obtains a faster convergence. Furthermore, in comparison to 4th-order algorithm, thirdorder one also obtains better results and its computational complexity is much lower. Therefore, 3rd-order cumulants lead to a faster noise reduction because of its higher aggressiveness with respect to both 4 th-order cumulants and autocorrelation function.

\section{THE INTERFRAME FACTOR}

In table 1, we may appreciate an improvement that increases gradually iteration by iteration. Most part of noise suppression is obtained after processing two iterations. Third-order cumulants obtain an appreciable noise suppression (about $2 \mathrm{~dB}$ in Cepstrum distance) after first iteration and then this speech modelling is enhanced a lot in the second iteration because it estimates Wiener filter from cleaner speech signal. At first iteration, speech AR modelling is computed from noisy signal without any initial information about the features of speech signal corresponding to the current frame. However, we know some information of the current speech frame by considering that speech signal features don't vary a lot between two consecutive overlapped frames. Therefore, we propose to obtain the first iteration $A R$ coefficients as a combination between current frame AR estimation (step 4) and previous frame AR coefficients. Thus, we dessign the non-causal Wiener filter (step 5) as a linear combination of coefficients $a_{k}$, belonging to two consecutive frames, calculated as follows:

$$
\begin{aligned}
& A_{k}(n, 1)=\text { IF } . a_{k}(n, 1)+(1-\text { IF }) . A_{k}(n-1, P F I) \\
& 0 \leq k \leq P ; 1 \leq P F I \leq \text { MAXITER } ; \quad 0 \leq I F \leq 1
\end{aligned}
$$

where $n$ is the current frame, PFI is the Previous Frame Iteration that we consider to help first iteration of the current frame and IF is the Interframe Factor. We write $a_{k}$ when coefficients are estimated directly from a noisy speech frame and we note capital letter $A_{k}$ when coefficients are coming from a linear combination of $a_{k}$. At the beginning of every speech activity we set parameter IF $=1$ because the information of last speech frame is not related to the current speech frame. It must be noted that it represents different frame lengths weather we are at the beginning of every speech activity or not. Wiener filter dessigns corresponding to the remaining iterations of the algorithm are estimated over a cleaner speech signal coming from Wiener filtering Output of previous iteration of the same frame:

$$
A_{k}(n, i t e r)=a_{k}(n, i t e r) \quad, 2 \leq \text { iter } \leq \text { MAXITER }
$$

We have two parametcrs to control this lincar combination. First parameter is the Interframe Factor IF that represents the amount of current speech $A R$ estimation $a_{k}(n, 1)$ we put in the AR modelling $A_{k}(n, 1)$ of the filter. The interframe factor is the main parameter to control linear combination (5) because parameter IF $=1$ represents that only current $A R$ estimation is considered to dessign Wiener filter at first iteration of current frame and then parameter PFI has no sense to be considered. Thus, parameter $\mathrm{IF}=1$ refers to a situation where no interframe factor is defined. If we decide to consider previous frame information (IF $<1$ ) we must consider parameter PFI to answer the following question : Which iteration number (PFI) of preceding frame must we take to obtain a reliable speech AR modelling? Preceding works [5],[6] have shown that it has no sense to process more than 5 iterations when third-order statistics are considered. Therefore, parameter MAXITER $=5$ has been fixed in all our tests.

On the other hand, parameter $I F=0$ represents that the coming noisy speech frame is filtered by means of a filter estimation coming from previous speech frames. Two different situations may be distinguished: $P F I=1$ and $P F I>1$. When information proceeding from first iteration of previous speech frame $(\mathrm{PFI}=1)$ is considered, no better results than before ( $\mathrm{IF}=1$ ) are expected, because the speech AR estimator is looking at the same noisy speech, but in a previous frame and performance therefore decreases when parameter IF decreases to 0 (see 1st iteration line in fig.3). However, a good improvement (about 1.5dB in Cepstrum distance) is obtained when parameter $\mathrm{PFI}>1$ but distortion effect increases more than $2 \mathrm{~dB}$ in Cepstrum distance (see fig.2) because current Wiener filter is dessigned with speech AR estimation proceeding from the preceding frame over a cleaner speech signal.

In fig.2, Cepstrum distance corresponding to first iteration of current frame has been represented and some different iteration numbers of preceding frame have been evaluated. Clean speech has been processed by this system and so distortion effect corresponding to the iterative algorithm has been depicted. To avoid an appreciable increase of distortion effect all values of parameter IF lower than 0.6 must be discarded. In fig. 3 , first iteration of current frame corresponding to speech signal disturbed by $A W G N$ at $S N R=0 \mathrm{~dB}$ has been processed and some different speech AR estimations of previous frame have been evaluated. We may come to the conclusion that values of parameter IF ranging from 0.6 to 0.8 represent a good tradeoff between distortion and noise suppression. Therefore, we may achieve an improvement of $2 \mathrm{db}$ in Cepstrum distance by introducing parameter $\mathrm{IF}(\mathrm{PFI}=3)$ to estimate current speech AR modelling without any noticeable increase of distortion. Thus, we may obtain an improvement higher than $4 \mathrm{~dB}$ in Cepstrum distance after processing only first iteration of the iterative Wiener filtering. In this way a good reduction of convergence speed is achieved and so a reduction of compulational complexity and processing delay are obtained by 
introducing the interframe factor IF and the previous frame iteration parameter PFI.

\section{CONCLUSIONS}

A speech enhancement method based on an iterative Wiener filtering have been proposed. Spectral estimation of speech is got by means of an AR modelling based on 3rd-order cumulant analysis to provide the desirable noisespeech uncoupling. Two parameters, IF (Interframe Factor) and PFI (Previous Frame Iteration), have been considered to take advantage of previous speech spectrum estimations to initiate AR modelling corresponding to

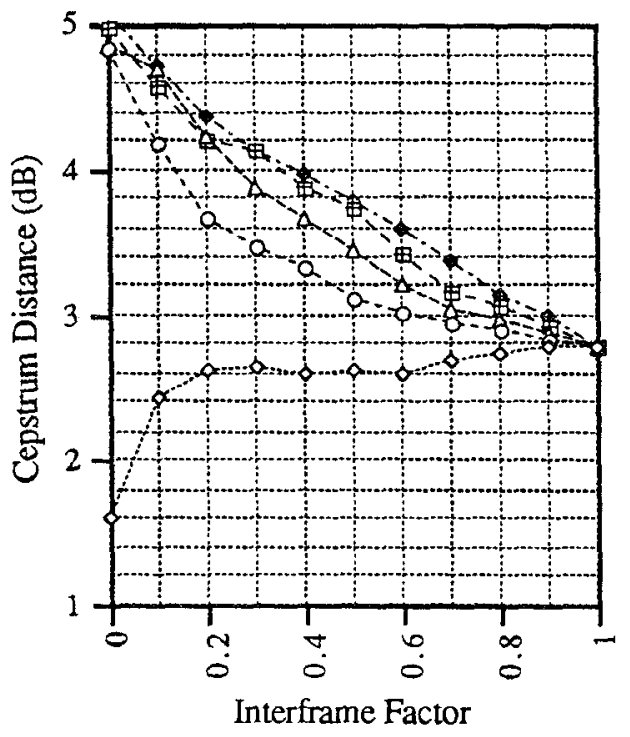

No iter. -

2nd iter. -...--... ---- $-\cdots-\cdots \quad$ 3rd iter

4 th iter. $-\cdots \oplus-\cdots \quad+\cdots \cdots$ 5th iter.

Fig.2: Distortion Effect introduced by first iteration processing when some different speech AR modellings, corresponding to different iterations of previous speech frame, are considered.

\section{REFERENCES}

[1]C.L. Nikias, M.R. Raghuveer."Bispectrum Estimation: A Digital Signal Processing Framework". Proc. of IEEE, pp 869-891. July 1987.

[2]J.S.Lim and A.V.Oppenheim."All-Pole Modeling of Degraded Speech". IEEE Trans ASSP, pp197-210.June 1978.

[3]J.H.L. Hansen and M.A. Clements."Constrained Iterative Speech Enhancement with Apllications to Spech Recognition". IEEE Trans on Sig. Proc., pp 795-805. April 1991.

14]E.Masgrau,J.M.Salavedra,A.Moreno,A.Ardanuy. "Speech Enhancement by Adaptive Wiencr Filtering based on Cumulant AR Modclling". Proc. ESCA Workshop on first iteration of the current speech frame. This approach achieves a noise suppression about $4 \mathrm{~dB}$ (Cepstrum Distance) after processing only first iteration of the algorithm. This fact represents an improvement about $2 \mathrm{~dB}$ (Cepstrum Distance) in relation to parameterized thirdorder algorithm ( $\mathrm{IF}=1)$. Finally, the convergence of the iterative algorithms based on cumulant AR estimation is strongly accelerated. Therefore, a good reduction of computational complexity and processing delay are achieved, while no appreciable increase of distortion effect is generated.

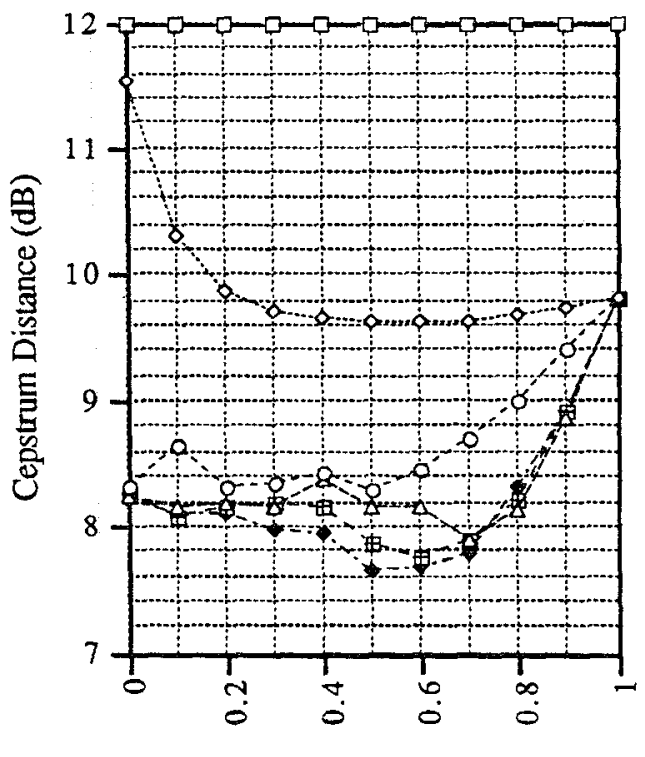

Interframe Factor

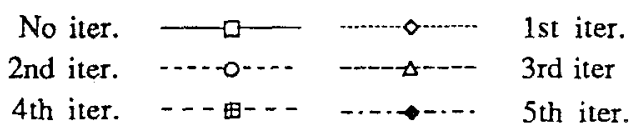

Fig.3: Noise Reduction after processing first iteration when some different speech AR modellings, corresponding to different iterations of previous speech frame, are considered.

Speech Processing in Adverse Conditions, pp 143-146. Cannes, France.November92.

[5]J.M.Salavedra,E.Masgrau,A.Moreno,X.Jové. "A Speech Enhancement System using HO AR estimation in real environments". Proc. EUROSPEECH'93, pp. 223-226. Berlin, Germany. September 21-23, 1993.

[6]J.M.Salavedra,E.Masgrau,A.Moreno,J.Estarellas, X.Jové. "Robust Coefficients of a HO AR Modelling in a Speech Enhancement System using parameterized Wiener Filtering". Proc. MELECON'94, pp. 69-72. Antalya, Turkey. April 12-14, 1994. 